\title{
Preferential Arrangement Superpatterns
}

\author{
Yonah Biers-Ariel ${ }^{1,2}$ \\ Department of Mathematics \\ Rutgers University \\ New Brunswick, NJ, USA \\ Anant Godbole ${ }^{1,4}$ \\ Department of Mathematics and Statistics \\ East Tennessee State University \\ Johnson City, TN, USA \\ Yiguang Zhang ${ }^{1,4}$ \\ Department of Applied Mathematics and Statistics \\ The Johns Hopkins University \\ Baltimore, MD, USA
}

\begin{abstract}
A superpattern is a string of characters of length $n$ that contains as a subsequence, and in a sense that depends on the context, all the smaller strings of length $k$ in a certain class. We prove structural and probabilistic results on superpatterns for preferential arrangements, including (i) a theorem that demonstrates that a string is a superpattern for all preferential arrangements if and only if it is a superpattern for all permutations; and (ii) a result that is reminiscent of a still unresolved conjecture of Alon on the smallest permutation on $[n]$ that contains all $k$-permutations with high probability.
\end{abstract}

Keywords: Superpattern; preferential arrangements; permutation. 


\section{Introduction and Statement of Results}

A superpattern is a string of characters of length $n$ that contains as a (not necessarily consecutive) subsequence, and in a sense that depends on the context, all the smaller strings of length $k$ in a certain class. Specifically, given a set $X$ and a class $\mathcal{R}$ such that each object in $\mathcal{R}$ is a string of $k$ elements in $X$, a superpattern is a string that contains all $p \in \mathcal{R}$ as subsequences. For example, with $X=\{1,2\}$ and $\mathcal{R}=\{11,12,21,22\}$,

is a superpattern.

In this paper, we present some results on superpatterns for preferential arrangements, or word-patterns. Key references in this area are [3], [5], and [7]. Preferential arrangements (p.a.'s) of length $k$ over $X=[d]:=\{1,2, \ldots, d\}$ are $k$-strings with entries from $[d]$, for which order isomorphic representations are considered to be equivalent. For example if $k=3, d=2$, there are seven preferential arrangements, viz. 111, 112, 121, 211, 122, 212, and 221. If $k=d=3$, the thirteen preferential arrangements (enumerated whenever $d=k$ by the ordered Bell numbers) are 111, 112, 121, 211, 122, 212, 221, and the six permutations $123,132,213,231,312$, and 321 . Note that, for example, the strings 112, 113, and 223 are order isomorphic, so above we just list the preferential arrangement 112, expressed in the traditional lexicographically minimal fashion, also known as a dense ranking system. If $k=3, d \geq 4$, there are still only 13 p.a.'s, since, for example, with $k=3$ and $d=4$, the six strings $112,113,114,223,224,334$ are each equivalent to the p.a. 112.

A superpattern for preferential arrangements of length $k$ over $[d]$ is an $n$ long string over the alphabet $[d]$, that contains, as a subsequence, each of the preferential arrangements of length $k$ over $[d]$ in any one of its order isomorphic forms. For example, a string such as 3213213 is a superpattern for $k=d=3$ or with $k=3 ; d=4$, and 1231241 is a superpattern with, e.g., $k=3 ; d=5$

\footnotetext{
$\overline{1}$ The first two authors were supported by NSF Grant 1263009. The third author was supported by the Acheson J. Duncan Fund for the Advancement of Research in Statistics

2 Email: ybiersariel@gmail.com

3 Email: godbolea@etsu.edu

${ }^{4}$ Email: yzhan132@jhu.edu
} 
or $k=3 ; d=4$. Let $n(k, d)$ be the length of the shortest superpattern for all p.a.'s of length $k$ over $[d]$.

The fact that $n(3,3)=7$ (tacitly used above) is elementary and has been shown, e.g., in [8] (in which the rather complicated waiting time distribution for a random string on $\{1,2,3\}$ to become a 3 -superpattern was also studied), and the authors of [3] conjectured that $n(k, k)=k^{2}-2 k+4$ for each $k$, a fact that we prove for $k=4$ in Section 2 of this paper. In the main result of Section 2, Theorem 2.4, we prove that for each $k, n(k, k)=\rho(k, k)=\rho(k)$, where $\rho(k)$ is the shortest superpattern with entries from the alphabet $\{1,2, \ldots, k\}$ that contains all the permutations of length $k$. This somewhat surprising result has ramifications: It was shown in [10] that the quantity $\left\lceil k^{2}-\frac{7}{3} k+\frac{19}{3}\right\rceil$, which is smaller than $k^{2}-2 k+14$ for $k \geq 10$, is an upper bound for $\rho(k)$. Since, via Theorem 2.4, $\rho(k)$ and $n(k, k)$ are the same, this disproves the $k^{2}-2 k+4$ conjecture.

The first paper to make the $k^{2}-2 k+4$ conjecture was [9], where this conjecture was presented as one of two. The other conjecture, which, at the present time appears to be the best candidate for the true value of $n(k, k)$, is

$$
n(k)=n(k, k)=k^{2}-m \cdot k+\sum_{i=1}^{m} i 2^{m-i} \text { for } 2^{m} \leq k \leq 2^{m+1}-1 .
$$

In the rest of the paper as well, we will focus on the case $k=d$. In Section 3, we generalize the work of [1] and [8] by exhibiting tight bounds on the expected waiting time until a random string, with each letter being independently and uniformly chosen from $[k]$, becomes a superpattern for $k$ long preferential arrangements (or permutations) over $[k]$. The waiting time is shown to be tightly concentrated around its mean. This result recalls the Alon Conjecture from [2], which states that the value $n=\frac{k^{2}}{4}$ is the threshold for a random permutation on $[n]$ to contain each of the $k$ ! $k$-permutations in an order isomorphic form - with high or low probability. In our result, the parent string contains repetitions, but the net result is still that each $k$-permutation must appear in a non-isomorphic form. As seen, e.g, in [4], Alon's conjecture is notoriously hard - but perhaps an approach exemplified by Theorem 2.4 might yield dividends.

\section{Length and Structure of Superpatterns}

In this section we will focus on the case $k=d$, and consider $n(k)$, the length of the shortest word on the alphabet $[k]$ containing all preferential arrangements 
of length $k$. We again consider $\rho(k)$, the length of the shortest word on the alphabet $[k]$ containing all permutations of the elements of $[k]$. We first observe that $n(k)=\rho(k)$ for $3 \leq k \leq 7$, and then show that $n(k)=\rho(k)$ for each $k$.

We begin by establishing a general lower bound for $\rho(k)$ which agrees with $k^{2}-2 k+4$ for $k=3,4$. Even though the coefficient on the leading term in Proposition 2.1 is $\frac{1}{2}$, making the bound asymptotically inferior to the bound $n(k) \geq k^{2}-c(\epsilon) k^{1.75+\epsilon}$ from [6], it suffices for small values of $k$ as we will see. Induction can be used to prove

Proposition $2.1 \rho(k) \geq \frac{k^{2}}{2}+\frac{3 k}{2}-2$ for all $k \geq 2$.

The fact that $\rho(k)=n(k)$ for $1 \leq k \leq 7$ (the 1 and 2 cases are trivial) suggests that this equality may hold for all $k$, and, in fact it does. Proving this, however, requires two new definitions. For Definitions 2.2.-2.3 and Theorem 2.4 , let $A_{k}=\left\{a_{1}, \ldots, a_{k}\right\}$ be an arbitrary subset of $\mathbb{N}$ with $\left|A_{k}\right|=k$ and $a_{1}<a_{2}<\ldots<a_{k}$.

Definition 2.2 A regular occurrence of a preferential arrangement in a word on the alphabet $A_{k}$ is an occurrence of that arrangement such that for each letter, supposing there are $i$ letters in the p.a. that are less than that letter and $j$ copies of that letter in the p.a., the letter is represented in the word by some element of $\left\{a_{i+1}, a_{i+2}, \ldots, a_{i+j}\right\}$.

For instance, if our alphabet is [6], then a regular occurrence of 112232 is one in which the 1's are represented by 1's or 2's, the 2's are represented by 3's, 4's, or 5's, and the 3 is represented by a 6. So, 113363 and 225565 are regular occurrences of 112232, but 113343 is not. Then, a regular superpattern of length $k$ p.a.'s on $[k]$ is defined to be a string that contains a regular occurrence of all p.a.'s.

Definition 2.3 A complete word on $A_{k}$ is a word on $A_{k}$ containing every permutation of the elements of $A_{k}$. So, $\rho(k)$ is the length of the shortest complete word on $[k]$.

Now, let $\mathcal{C}_{A_{k}}$ be the set of complete words on $A_{k}$, let $\mathcal{S}_{A_{k}}$ be the set of superpatterns of length $k$ preferential arrangements on $A_{k}$, and let $\mathcal{R}_{A_{k}}$ be the set of regular superpatterns of length $k$ preferential arrangements on $A_{k}$.

Theorem 2.4 For all $A_{k}$ with $k \geq 2, \mathcal{C}_{A_{k}}=\mathcal{S}_{A_{k}}=\mathcal{R}_{A_{k}}$.

Theorem 2.4, proved by induction, is useful in two regards. First, it allows us to apply everything known about complete words to superpatterns of preferential arrangments. As noted in the introduction, this immediately gives us 
that $\left\lceil k^{2}-\frac{7}{3} k+\frac{19}{3}\right\rceil$ is an upper bound on $n(k)$, thereby disproving a longstanding conjecture. Theorem 2.4 could also potentially help in finding lower bounds for $n(k)$ because proving that no words of a certain length are regular superpatterns may be easier than proving that no words are superpatterns, but this approach has not been fruitful so far.

\section{Random Superpatterns}

Finally, we will prove a result regarding random superpatterns. Consider the following random process: beginning with an empty word $\bar{W}$, at each timestep we choose a letter, uniformly at random, from the alphabet $[k]$. We then concatenate this value onto the end of $\bar{W}$ and check to see if the augmented $\bar{W}$ is a superpattern for all $k$-long preferential arrangements on $[k]$ (or, equivalently, a complete word on $[k]$ ). We are interested in the value of $E\left[X_{k}\right]$ where $X_{k}$ is the first timestep at which $\bar{W}$ is a superpattern on $[k]$. This problem was first considered by Godbole and Liendo in [8]. There, the authors found values for $E\left[X_{2}\right]$ and $E\left[X_{3}\right]$ as well as the exact distributions of $X_{2}$ and $X_{3}$; here we will apply a previous result to give a general upper bound on $E\left[X_{k}\right]$, and then prove an lower bound. These bounds will be asymptotically equivalent and together prove that $E\left[X_{k}\right] \sim k^{2} \log k$. The distribution of $X_{k}$ appears to be intractable for $k \geq 4$, and a good agenda for the future, as pointed out by a referee, might be to model the above as the hitting time in some finite Markov chain.

Abraham et al. consider a similar problem in [1]; they were interested in omnisequences which must contain every $k$-letter word on $[k]$, and find that $Z_{k}$, the expected number of randomly chosen letters necessary to produce an omnisequence is asymptotically $k^{2} \log k$ (with error terms as described below). Since every omnisequence of $k$-letter words on $[k]$ is also a superpattern on $[k]$, we obtain from Abraham's work that $E\left(X_{k}\right) \leq k^{2}\left(\log (k)+\gamma+O\left(k^{-1}\right)\right)$ for all $k$, where $\gamma \approx .577$ is Euler's constant and $\log$ denotes the natural logarithm. The next theorem provides a similar lower bound.

Theorem 3.1 For all $k, E\left[X_{k}\right] \geq k^{2}\left(\log (k)+\gamma-1+O\left(k^{-1}\right)\right)$.

A conjecture of Noga Alon states that for a random permutation of $[n]$ to contain all $k$ ! permutations of $[k]$ with high probability, one must have $n=\frac{k^{2}}{4}(1+o(1))[2]$. While this conjecture has remained open for fifteen years, we will prove an analogue regarding superpatterns of preferential arrangements (equivalently superpatterns of permutations) when restricted to the alphabet $[k]$ by showing that $X_{k}$ is highly concentrated about its mean. 
Theorem 3.2 With high probability, we have that $k^{2} \log k-(1-\gamma) k^{2}-$ $\omega(1) k^{\frac{3}{2}} \leq X_{k} \leq k^{2} \log k+\gamma k^{2}+\omega(1) k^{\frac{3}{2}}$ where $\omega(1)$ is any sequence tending to $\infty$.

\section{References}

[1] S. Abraham, G. Brockman, A. Godbole, and S. Sapp, Omnibus sequences, coupon collection, and missing word counts, Methodology and Computing in Applied Probability 15 (2013), 363-378.

[2] R. Arratia. On the Stanley-Wilf Conjecture for the Number of Permutations Avoiding a Given Pattern, Electr. J. Combinatorics 6 (1999), Paper N1.

[3] A. Burstein, P. Hästö, T. Mansour. Packing Patterns into Words, Electr. J. Combinatorics 9 (2002-03), Paper R20.

[4] H. Eriksson, K. Eriksson, S. Linusson, and J. Wästlund. Dense packing of patterns in a permutation, Ann. Combinatorics 11 (2007), 459-470.

[5] A. Evdokimov, V. Nyu. Length of a supersequence for a set of binary words with a given number of units, Metody Diskretnogo Analiza 52 (1992), 49-58, in Russian.

[6] D. J. Kleitman and D. J. Kwiatkowski. A lower bound on the length of a sequence containing all permutations as subsequences, Journal of Combinatorial Theory, Series A 21 (1976), 129-136.

[7] L. Kontsevich. Uniform placements, Kvant 7 (1985), 51-52, in Russian.

[8] M. Liendo and A. Godbole. Waiting Time Distribution for the Emergence of Superpatterns, Methodology and Computing in Applied Probability DOI: 10.1007/s11009-015-9439-6 (2016+).

[9] M. Newey. Notes on a problem involving permutations as subsequences, Technical Report, Stanford University (1973).

[10] S. Radomirović. A construction of short sequences containing all permutations of a set as subsequences, Electr. J. Combinatorics 19 (2012), Paper P31. 